Le partenariat pour la formation des formateurs à l'écocitoyenneté - Exploration d'une problématique

Yannick Bruxelle, Jean Burger et Francis Thubé

\author{
(2) OpenEdition \\ Journals \\ Édition électronique \\ URL : https://journals.openedition.org/ere/7225 \\ DOI : $10.4000 /$ ere.7225 \\ ISSN : 2561-2271 \\ Éditeur \\ Centr'ERE
}

Référence électronique

Yannick Bruxelle, Jean Burger et Francis Thubé, «Le partenariat pour la formation des formateurs à l'écocitoyenneté - Exploration d'une problématique», Éducation relative à l'environnement [En ligne], Volume 1 | 1999, mis en ligne le 15 septembre 1999, consulté le 28 mai 2021. URL : http:// journals.openedition.org/ere/7225; DOI : https://doi.org/10.4000/ere.7225 


\title{
Le partenariat pour la formation des formateurs à l'écocitoyenneté - Exploration d'une problématique
}

\author{
Yannick Bruxelle, Jean Burger et Francis Thubé
}

1 Nous souhaitons, à travers cet exposé, d'une part témoigner des pratiques de partenariat que nous mettons en œuvre pour organiser et vivre des formations relevant de l'Éducation nationale française et d'autre part traduire l'état de notre réflexion sur le partenariat en éducation relative à l'environnement (ERE).

2 En 1993, lors de la signature du deuxième protocole entre le ministère de l'Environnement et le ministère de l'Éducation nationale, l'Académie ${ }^{1}$ de Poitiers a été désignée l'une des quatre académies pilotes pour la formation en éducation à l'environnement. Notre équipe de formateurs a l'originalité d'être constituée à la fois de personnes relevant du ministère de l'Éducation nationale (Action culturelle du Rectorat $^{2}$ et Institut Universitaire de Formation des Maîtres) et de personnes relevant d'associations ou de structures régionales (Groupe régional d'animation et d'initiation à la nature et à l'environnement en Poitou-Charentes - GRAINE du réseau École et Nature, Parc Interrégional du Marais Poitevin et depuis peu, l'Institut de formation et de recherche en éducation à l'environnement - Ifrée $\left.{ }^{3}\right)$. Elle pratique ce partenariat depuis une dizaine d'années.

3 Dès le départ, cette équipe s'est bien inscrite dans une démarche de recherche-action et même si elle n'a pas encore actuellement associé de chercheurs en sciences de l'éducation, elle analyse ses pratiques au fil des années et cherche à avoir un regard critique sur ses actions. Ainsi, chaque stage est l'objet d'évaluations très serrées, faites sans complaisance, qui servent de base pour la mise en place des stages suivants.

4 Avec la création de l'Ifrée en Poitou-Charentes, cette démarche se formalise encore plus à travers le processus d'écriture de documents plus précis au sein desquels nous cherchons, à partir de nos expériences, à identifier des éléments transférables à d'autres situations et à faire part de l'état d'avancement de notre réflexion collective. 
5 Les temps de conception, d'organisation et d'encadrement d'un stage national (Plan National de la Direction des Écoles), tel que celui intitulé L'écocitoyenneté: l'homme dans le village, l'homme dans la ville (collectif, 1997) et présenté en annexe, sont pour l'équipe des moments particulièrement forts. Compte tenu des spécificités du public (ici, des formateurs de l'Éducation nationale: professeurs des Instituts Universitaires de Formation des Maîtres, inspecteurs, conseillers pédagogiques, maîtres formateurs et des formateurs issus du monde associatif : centres de découverte, centres permanents d'initiation à l'environnement, etc.), chaque partenaire formateur formule les objectifs qu'il se fixe et c'est à partir de l'ensemble de ces objectifs que des propositions sont faites et que des choix sont négociés.

6 Ainsi, en ce qui concerne la structure d'accueil, le Centre de découverte d'Aubeterre a une longue pratique de l'accueil des classes environnement et des stages de formation pour adultes. L'aménagement des locaux, les choix pédagogiques de l'équipe (jusque dans les petits plats de la cuisinière) reflètent une volonté de créer les conditions idéales pour la formation et la réflexion sur l'environnement et le développement durable.

7 De même, pour la démarche, le choix méthodologique qui a été fait pour la conception du stage correspond aux valeurs portées par l'équipe d'organisation. Ce choix s'appuie sur le partenariat et la confrontation des points de vue et des pratiques, dans le cadre d'une approche humaniste.

Cette démarche croise une pédagogie active au service de l'éducation environnementale, démarche de projet incluant un travail sur les représentations, une phase d'immersion basée sur le sensoriel - sensible, une phase de recherche active favorisant les questionnements et tendant vers une vision systémique avec une pédagogie de la responsabilisation de chaque personne : écoute, prise en compte des points de vue des autres, confrontation (notamment lors des approches et regards croisés) qui favorise le cheminement personnel de chacun au travers des échanges, apports et réflexions. L'équipe partenariale se situe plutôt en termes d'accompagnement de la réflexion des stagiaires sur ce terme nouveau et sans doute provisoire d'écocitoyenneté en favorisant les moments de réflexion, mais en précisant toujours qu'elle ne détient pas la bonne définition!

9 Le mot " partenariat » est relativement nouveau puisqu'il apparaît seulement en 1987 dans notre dictionnaire français; et, comme le montre Corinne Mérini (1995), son étymologie et son histoire sont à l'image de sa complexité. Ainsi, il peut sembler être lié à un effet de mode, et il nous apparaît nécessaire de bien clarifier comment chacun définit le « partenariat ».

$10 \mathrm{Au}$ sein du ministère de l'Éducation nationale en France, existe un cadre réglementaire qui autorise, favorise et officialise le partenariat: c'est « l'action culturelle en milieu scolaire" qui a des représentants au sein de chaque rectorat. Toutefois, malgré les directives nationales, on constate que selon les académies, ce secteur est plus ou moins développé en fonction de la volonté des responsables successifs et surtout que tous ces services, selon l'implication personnelle de leurs acteurs de terrain, n'ont pas la même conception du partenariat.

11 De façon générale, on considère que sont partenaires ceux qui « apportent leur part » à un projet, celle-ci pouvant être financière, technique et, ou pédagogique. Pour nous, sont vraiment partenaires ceux qui cheminent réellement ensemble du début du projet 
jusqu'à son évaluation. Ceux qui ne font qu'une partie du chemin sont plutôt des intervenants qui participent au projet dans le cadre d'une prestation de service.

La relation de partenariat implique donc que les partenaires négocient ensemble les points de croisement de leurs objectifs au travers d'une action commune qui, si le partenariat a bien été réel, sera forcément différente de l'idée que chacun pouvait s'en faire au départ. Chaque partenaire devra être clairement identifié pour pouvoir se reconnaître dans un projet commun et en être fier.

Pour nous, la relation entre partenaires est forcément horizontale et égalitaire, sans assujettissement, même si un coordinateur de projet est indispensable et même si, dans le contexte qui nous concerne, au bout du compte, c'est forcément l'Éducation nationale qui porte officiellement la responsabilité pédagogique de l'ensemble du stage. Si une relation verticale de subordination s'instaurait, on ne parlerait plus de partenariat, mais de parrainage.

Nous pensons qu'une réelle pratique du partenariat telle que décrite ci-dessus, est en mesure de modifier profondément les systèmes dont nous relevons car :

- elle n'est pas sans effet sur les personnes qui pratiquent alors un réel exercice de citoyenneté (esprit d'ouverture, respect de l'autre, acceptation des différences, etc.) et ont souvent le sentiment de rompre avec des schémas classiques;

- elle n'est pas sans effet sur les sujets traités qui bénéficient ainsi des points de vue différents, des compétences complémentaires et des richesses des partenaires ;

- elle n'est pas non plus sans effet sur les institutions qui sont amenées à s'ouvrir, à évoluer, à reconnaître que différentes dynamiques concourent vers une même finalité.

Car, que ce soit dans la société civile, dans les entreprises ou dans l'administration, qui peut prétendre réunir l'ensemble des compétences pour parler d'environnement et à fortiori d'écocitoyenneté ? Seules la rencontre des acteurs, la confrontation des regards et l'écoute réciproque peuvent permettre de poser les bonnes questions pour tenter ensuite de les résoudre. Les "spécialistes » de l'environnement doivent donc avant tout, être des médiateurs. Il n'en est pas autrement au sein de l'école où la formation à l'environnement et à l'écocitoyenneté se doit de favoriser, au plus tôt, ces aptitudes à communiquer et à travailler en équipe.

Nous avons pour notre part, en Poitou-Charentes, acquis une certaine expérience de la pratique partenariale qui nous a permis d'entamer cette réflexion évoluant et s'enrichissant au fil des formations. Un ouvrage intitulé Écocitoyenneté: pratiques et démarches pour la formation (collectif, en cours), prenant appui sur plusieurs expériences, paraîtra sous peu. Les coauteurs y précisent le pourquoi de leur attachement à ce thème de l'écocitoyenneté, mais aussi, en s'appuyant sur leurs pratiques et sur des recherches notamment en sciences de l'éducation, cherchent à mettre en évidence les conditions favorables à réunir pour une éducation relative à l'environnement de qualité. Les difficultés du partenariat sont grandes; c'est une pratique dévoreuse de temps et les prises de pouvoir ou au contraire la délégation excessive sont tentantes. Mais au regard du plaisir de l'échange et de la rechercheaction collective et surtout des enjeux pour la formation des futurs citoyens, la balance semble bien pencher en sa faveur. Des pistes de recherche-action sont ouvertes, etc., notamment grâce à une démarche de rapprochement avec d'autres académies de France afin de mutualiser nos expériences et nos réflexions. 


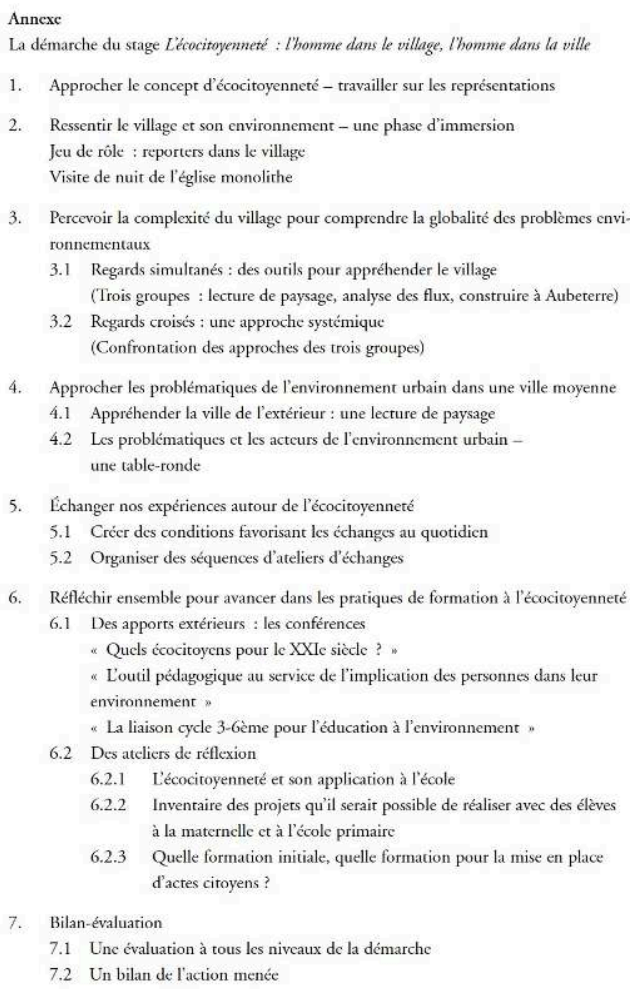

\section{BIBLIOGRAPHIE}

Collectif. (en cours). Écocitoyenneté : pratiques et démarches pour la formation. PoitouCharentes : Ifrée.

Collectif. (1997). L'écocitoyenneté : l'homme dans le village, l'homme dans la ville. Actes du stage Plan National de Formation, 9-14 décembre 1996. Poitou-Charentes : Ifrée.

Mérini, C. (1995). Le partenariat, un nouveau mode d'action ? Signification et modélisation. Communication présentée dans le cadre d'une Université d'été, 1000 défis pour l'Éducation à l'Environnement, septembre 1995, Rambouillet, France.

\section{NOTES}

1. Le ministère de l'Éducation nationale découpe le territoire français en académies qui regroupent de 2 à 5 départements et correspondent parfois aux régions administratives (c'est le cas pour l'Académie de Poitiers et la région Poitou-Charentes).

2. Dans chaque académie, le ministre de l'Éducation nationale nomme son représentant appelé un recteur d'académie. Celui-ci est responsable de l'ensemble des services administratifs et pédagogiques constituant le rectorat ; il est par ailleurs chancelier des universités.

3. L'Ifrée existe depuis 3 ans, ses missions se déclinent en 4 axes: 1) la formation des acteurs et des médiateurs de l'éducation à l'environnement; 2) la mise au point et la diffusion de 
documents et d'outils pédagogiques ; 3) la recherche en éducation à l'environnement en particulier sur la question de l'évaluation des actions ; 4) l'observation de l'environnement en développant l'observatoire régional de l'environnement.

\section{AUTEURS}

\section{YANNICK BRUXELLE}

Yannick Bruxelle est professeure certifiée de sciences naturelles, détachée au Rectorat de l'Académie de Poitiers à la Mission d'action culturelle pour susciter le développement de projets interdisciplinaires et partenariaux avec les élèves dans les établissements scolaires. Ses missions concernent les secteurs de l'éducation à l'environnement et de la culture scientifique et technique et intègrent des actions de formation continue.

\section{JEAN BURGER}

Jean Burger est directeur de l'Ifrée (Institut de formation et de recherche en éducation à l'environnement) en Poitou-Charentes. Après avoir été enseignant, il travaille comme animateur et formateur en éducation à l'environnement depuis une vingtaine d'années.

\section{FRANCIS THUBÉ}

Francis Thubé est directeur du GRAINE (Groupe régional d'animation et d'initiation à la nature et à l'environnement) de Poitou-Charentes, vice-président de l'Ifrée et administrateur du réseau national français École et Nature. Animateur de terrain, formateur de formateurs et gestionnaire de projets liés à l'environnement et à la citoyenneté, il travaille dans un souci constant de mise en œuvre de partenariat basé sur l'action. 